\title{
Tailoring Disorder and Quality of Photonic Glass Templates for Structural Coloration by Particle Charge Interactions
}

Yen Häntsch, Guoliang Shang, Bo Lei, Benedikt Winhard, Alexander Petrov, Manfred Eich, Elizabeth Holm, Gerold A. Schneider, and Kaline P. Furlan*

Cite This: ACS Appl. Mater. Interfaces 2021, 13, 20511-20523

Read Online

ABSTRACT: To obtain high-quality homogeneous photonic glass-based structural color films over large areas, it is essential to precisely control the degree of disorder of the spherical particles used and reduce the crack density within the films as much as possible. To tailor the disorder and quality of photonic glasses, a heteroaggregation-based process was developed by employing two oppositely charged equal-sized polystyrene (PS) particle types. The influence of the particle size ratio on the extent of heteroaggregation in the suspension mixes is investigated and correlated with both the morphology and the resultant optical properties of the films. The results show that the oppositely charged particle size ratio within the mix greatly influences the assembled

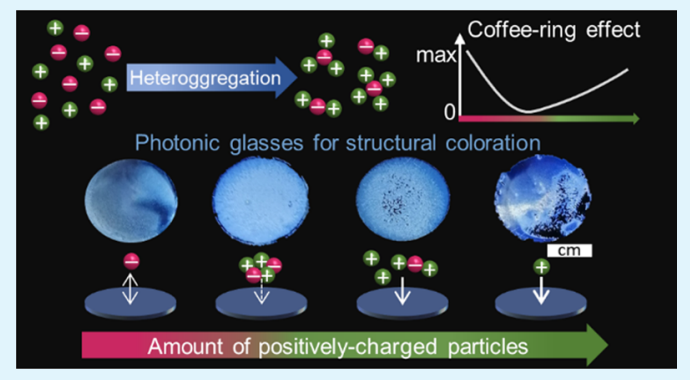
structure in the films, affecting their roughness, crack density, and the coffee-

ring formation. To better differentiate the morphology of the films, scanning electron microscopy images of the microstructures were classified by a supervised training of a deep convolutional neural network model to find distinctions that are inaccessible by conventional image analysis methods. Selected compositions were then infiltrated with $\mathrm{TiO}_{2}$ via atomic layer deposition, and after removal of the PS spheres, surface-templated inverse photonic glasses were obtained. Different color impressions and optical properties were obtained depending on the heteroaggregation level and thus the quality of the resultant films. The best results regarding the stability of the films and suppression of coffee-ring formation are obtained with a 35 wt \% positively charged over negatively charged particle mix, which yielded enhanced structural coloration associated with improved film quality, tailored by the heteroaggregation fabrication process.

KEYWORDS: photonic glasses, self-assembly, heteroaggregation, structural color, high-temperature photonics

\section{INTRODUCTION}

Photonic crystals and glasses are structured materials with a known capability of affecting the propagation and, for example, the reflection of electromagnetic radiation depending on the structural parameters, material(s) refractive index/indices, and wavelength of light. Photonic glasses, disordered arrangements of spherical particles, are particularly interesting, as they provide direction-independent spectral selectivity. The application of such disordered spectrally selective materials ranges from thermophotovoltaic energy conversion devices and reflective thermal barrier coatings to random lasing and noniridescent structural colors. ${ }^{1-5}$ The effect of such a structure on the reflection of light will depend directly on the $3 \mathrm{D}$ structural morphology and, therefore, is entirely linked to the fabrication process.

In the past, researchers have developed different methods to generate photonic glasses, each having its distinguished characteristics and resulting in structures with different scattering efficiencies. The concept of photonic glass was introduced by Ballato et al. ${ }^{6}$ in 1999 who used centrifugal forces to obtain disordered structures. In 2007, García et al. developed a simple drop-cast method to produce polystyrene
(PS)-based structures. ${ }^{7,8}$ The same method was used by Espinha et al. ${ }^{9}$ to produce PS-based photonic glasses. However, their method involves use of calcium chloride salt to cause flocculation of the colloidal suspensions (Figure 1a). After solvent evaporation, the salt is left behind on the structure, which may influence the optical properties. Additionally, further steps to remove the precipitated salt may be necessary if the structure is used as a template for further material infiltration.

The addition of extra compounds was also the approach chosen by Eurov et al. ${ }^{10}$ when studying the coagulation of $\mathrm{SiO}_{2}$-water colloidal suspensions via modifications on the solvent composition with the addition of ammonium chloride, hydrochloric acid, or cetrimonium bromide. In this case, the extra compounds could be removed by thermal annealing,

Received: January 21, 2021

Accepted: April 2, 2021

Published: April 20, 2021 

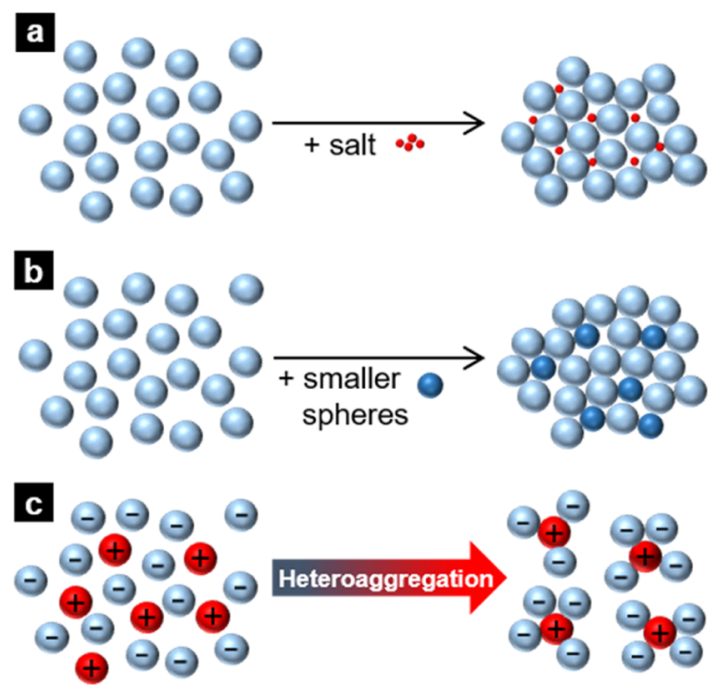

Figure 1. Scheme showing different approaches to introduce disorder into colloidal systems to fabricate photonic glasses by (a) using salt or (b) smaller spheres as additives or (c) heteroaggregation of monodisperse spheres with opposite charges. In $(a, b)$, either the additives remain in the structure after fabrication or particles with different sizes are used, while in (c), the particles have the same size and no additives are added.

which is possible for the $\mathrm{SiO}_{2}$ particles that can withstand higher temperatures but not for polymeric particle structures usually based on PS or polymethylmethacrylate particles.

Silica-based photonic glasses without the addition of extra compounds were presented by the Takeoka group ${ }^{11}$ in 2010 employing the concept of mixing two different $\mathrm{SiO}_{2}$ particle sizes, 310 and $220 \mathrm{~nm}$, as indicated in Figure $1 \mathrm{~b}$. The addition of the smaller particles disturbed the particle self-assembly during evaporation, resulting in an amorphous structure with a short-range order. Different structural colors were produced by this approach and showed angle independency for the studied viewing angles ranging from 0 to $40^{\circ}$.

A similar approach was used by Kim et al., ${ }^{12}$ but in this case, $\mathrm{SiO}_{2}$-PS core-shell particles with diameters of 242 and 268 $\mathrm{nm}$ were used for template assembly, while the inverted structure was generated by infiltrating the template with a photopolymer (trimethylolpropane ethoxylate triacrylate) and burning out of the PS particles. To produce the structural coloration, carbon nanotubes (CNTs) were added to the structure to act as a black absorber and suppress multiple scattering. The produced inverse photonic glasses with CNT additions showed green and red colors with angle independency from 0 to $60^{\circ}$.

In a similar manner, Forster et al. ${ }^{13}$ also explored the addition of a black absorber together with bimodal particles (226 and $271 \mathrm{~nm}$ ), although in this case, the black absorber particles were added prior to the assembly. For this reason, it is not clear whether the formation of photonic glasses was caused by the bimodal distribution only or also by the interaction with the carbon black particles, which could hinder the assembly of ordered structures, as demonstrated by Zhang et al. for a PScuttlefish ink particle system. ${ }^{14}$ The disturbance induced by the absorber particles could be caused either by the size and shape difference or by the heterocoagulation due to different surface charges. The introduction of black absorbers in the form of nanoparticles generally increases the surface roughness of photonic glasses, which results in matte structural colors. ${ }^{15}$
As a matter of fact, heterocoagulation has been widely used to generate hollow-sphere photonic glasses ${ }^{16}$ or, in several publications from the 2000s, the so-called macroporous structures. ${ }^{17-24}$ One has to distinguish, however, between the production of macroporous materials and hollow-sphere photonic glasses. Although a hollow-sphere photonic glass is considered to be a macroporous material, not all macroporous materials will be an effective photonic glass, as demonstrated by do Rosário et al. ${ }^{16}$

Moreover, earlier works on heterocoagulation were based on the interaction between particles with different surface charges (usually positive-negative charge pair) and also with very different sizes. For instance, the particle size ratios used in the works by do Rosário et al., ${ }^{16}$ Jia et al., ${ }^{17}$ and Tang et al. ${ }^{22}$ were approximately $75: 1,13: 1$, and 6:1, respectively. A successful heterocoagulation is based on the assumption that the smaller particles assemble in the form of a shell around the bigger particles, $^{25}$ forming a raspberry-like shape ${ }^{26}$ and, in an ideal case, leading to a total coverage of the bigger particle surface.

Lee et al. ${ }^{1}$ has shown that surface-templated opals produced by, for example, sol-gel or atomic layer deposition (ALD) can outperform other opals such as the hollow-sphere ones (usually generated by heterocoagulation) or the full sphere ones in which the so-called template would then already be the final structure. Moreover, the authors revealed that the degree of infiltration of the structure indicated by the core-shell ratio (air-material) directly influences the maximum photonic gap width $\Delta \omega / \omega$. Furthermore, infiltration of polymeric templates broadens the material selection possibility and enables the fabrication of a variety of ceramic materials. Although fabrication of monodisperse ceramic particles is possible, ${ }^{27}$ it is rather difficult to produce batches with low dispersity, and usually, a smaller yield is achieved in comparison to polymeric particles. There is, therefore, a great interest in generating inverse structures via ALD infiltration of polymeric templates. ALD is a deposition technique capable of conformal coatings with compositional tuning options and film thickness control on the Angstrom scale. ALD is also capable of generating ceramic-based photonic materials envisaged for high-temperature applications, such as titania, ${ }^{28}$ alumina, ${ }^{29}$ and mullite. ${ }^{30}$

Moreover, if an inverse structure is desired, the template particles have to be removed after the infiltration step. This is easily performed in polymeric templates by thermal burnout, while for $\mathrm{SiO}_{2}$-based templates, an etching step with hydrofluoric acid solution is needed, which could limit the choice of materials for the inverted structure. On account of that, in this work, we have focused on polymeric templates rather than the $\mathrm{SiO}_{2}$-based ones. We also utilized only single particle size for the template, as opposed to the polymer-based photonic glasses generated via the bimodal particle approach. ${ }^{11-13,31}$ For the bimodal particle route, one has to carefully choose the number ratio between the particles such that a disordered structure is formed and not a binary crystal. $^{31,32}$ This is not trivial, and the ratio depends on and varies with the particle size, ${ }^{11,13}$ giving different color impressions and $\lambda_{\max }$ depending on the number and size ratio. ${ }^{11,12} \lambda_{\max }$ stands for the wavelength in which the highest reflectivity is achieved. Lee et al. ${ }^{32}$ have recently demonstrated experimentally that the degree of colloidal ordering is directly related to the bimodal particle ratio and therefore directly influences the photonic response.

Nevertheless, the analysis of the results provided by the earlier works on heterocoagulation, the more recent works on 
bimodal particles, and the work by Lin et al. ${ }^{33}$ where heteroaggregation rate constants were measured brought us the idea of a facile and straightforward process to produce high-quality photonic glass templates. In this work, we developed a novel method to fabricate additive-free photonic glasses by controlled heteroaggregation and sedimentation of unimodal PS particle mixes. We used the commercially available water-based monodisperse PS particle suspensions and confined drop casting as an assembly method. Heteroaggregation was promoted by the surface charge difference of the equal-sized PS particles, namely, strongly negative and positive (Figure 1c). The influence of addition of positively charged PS particles for strongly negative pairs was investigated without any additives or surfactants. The formation and size of agglomerates, which depend on the mixing fraction, were characterized via combined dynamic light scattering and zeta potential measurements. The combination of PS with different surface charges effectively formed agglomerates for all mixing conditions. We observed that for each mix, the agglomerate formation rate versus the sedimentation rate varied, which directly influenced the solvent evaporation rate. Furthermore, the proposed method was effective in inhibiting the coffee-ring effect, ${ }^{34}$ as well as crack formation, depending on the mix condition. We show that a combination of adequate agglomerate size and sedimentation rate is crucial for the final properties of the photonic glasses, which were assessed by optical and scanning electron microscopy (SEM), image analysis with machine learning methods, profilometry, and reflectance measurements.

\section{MATERIALS AND METHODS}

Water-based monodisperse colloidal suspensions of PS particles with a $200 \mathrm{~nm}$ nominal diameter were purchased from Micromod Partikeltechnologie GmbH (Germany) and used as received with a concentration of $25 \mathrm{mg} / \mathrm{mL}$. Two types of particle surfaces were compared and studied, plain, indicating no surface modification after particle synthesis and therefore the negative terminated surface due to sulfate groups originating from the polymerization initiator compound, and surface-modified particles with trialkylammonium $\left(\mathrm{NR}_{3}{ }^{+}\right)$ groups. The zeta potential of the starting colloidal suspensions of plain and $\mathrm{NR}_{3}{ }^{+}$-functionalized PS (see Figure $\mathrm{S} 1$ ) over a $\mathrm{pH}$ range of 2-9 was provided by the particle supplier using a Zetasizer Nano ZS (Malvern Instruments).

The flocculation behavior of the suspension mixes was studied by keeping the total suspension volume fixed to $3 \mathrm{~mL}$ and varying the amount of $\mathrm{NR}_{3}{ }^{+}$(always positive)-functionalized particles added on top of the plain (always negative) particles. Pristine suspensions were also characterized to assess their behavior in relation to the substrate (sapphire) and as a comparative guide to the mixes. A summary of the prepared suspensions can be visualized in Table 1. After agitation, the suspensions were directly used to produce photonic structures and also diluted with ultrapure water to $0.15 \mathrm{mg} / \mathrm{mL}$ for zeta potential and size distribution measurements (Zetasizer Nano ZS, Malvern Instruments).

Photonic crystals and glasses were fabricated by drop-casting 200 $\mu \mathrm{L}$ of each suspension on top of the previously cleaned sapphire substrates $(\langle 1 \overline{1} 02\rangle, 30 \mathrm{~mm} \times 25 \mathrm{~mm}$, Crystec $\mathrm{GmbH})$. Hydrophobic rings (inner $\varnothing 20 \mathrm{~mm}$ ) made out of polytetrafluoroethylene were clamped to the substrates to confine the suspensions, avoid spreading, and assuring that all the produced photonic structures had the same area. The cleaning procedure of the substrates involved ultrasonication for $1 \mathrm{~h}$ in a $1 \mathrm{vol} \%$ solution of Mucasol, an alkalinebased detergent (Merz Hygiene GmbH, Germany), brushing, rinsing with ultrapure water, and drying under a $\mathrm{N}_{2}$ flow. The substrates were then further treated via $20 \mathrm{~min}$ exposure to an oxygen plasma
Table 1. Description of the Particle Mixes

\begin{tabular}{|c|c|c|c|c|}
\hline \multirow[b]{2}{*}{$\operatorname{mix} \#$} & \multicolumn{2}{|c|}{ mix description } & \multirow[b]{2}{*}{ weight $\%$ of plain } & \multirow[b]{2}{*}{ weight $\%$ of $\mathrm{NR}_{3}{ }^{+}$} \\
\hline & base & added & & \\
\hline 1 & plain & none & 100 & \\
\hline 2 & plain & 5 wt $\% \mathrm{NR}_{3}^{+}$ & 95 & 5 \\
\hline 3 & plain & 10 wt $\% \mathrm{NR}_{3}^{+}$ & 90 & 10 \\
\hline 4 & plain & 20 wt $\% \mathrm{NR}_{3}^{+}$ & 80 & 20 \\
\hline 5 & plain & 30 wt $\% \mathrm{NR}_{3}^{+}$ & 70 & 30 \\
\hline 6 & plain & 35 wt $\% \mathrm{NR}_{3}^{+}$ & 65 & 35 \\
\hline 7 & plain & 40 wt $\% \mathrm{NR}_{3}^{+}$ & 60 & 40 \\
\hline 8 & plain & 50 wt $\% \mathrm{NR}_{3}^{+}$ & 50 & 50 \\
\hline 9 & plain & 60 wt $\% \mathrm{NR}_{3}{ }^{+}$ & 40 & 60 \\
\hline 10 & plain & 80 wt $\% \mathrm{NR}_{3}{ }^{+}$ & 20 & 80 \\
\hline 11 & plain & 90 wt $\% \mathrm{NR}_{3}^{+}$ & 10 & 90 \\
\hline 12 & plain & 95 wt $\% \mathrm{NR}_{3}^{+}$ & 5 & 95 \\
\hline 13 & $\mathrm{NR}_{3}^{+}$ & none & & 100 \\
\hline
\end{tabular}

(Polaron PT7160, Quorum Technologies). After drop-casting, the samples were left at room temperature in ambient conditions for $24 \mathrm{~h}$.

Atomic layer deposition (ALD) was used to infiltrate the selected photonic glass templates with titanium dioxide to enhance their color saturation, as demonstrated in our recent work. ${ }^{35}$ The cycles were performed at $95{ }^{\circ} \mathrm{C}$ to avoid template decomposition or deformation and under a constant flow of nitrogen $(15 \mathrm{sccm})$. An in-house custom reactor (Hamburg University of Technology, Germany) was used and operated under the exposure mode for 300 cycles of titanium isopropoxide (TTIP, Sigma-Aldrich, heated to $85{ }^{\circ} \mathrm{C}$ ) and deionized water $\left(\mathrm{diH}_{2} \mathrm{O}\right)$. Pulse, exposure, and pump times (in seconds) were $1 / 30 / 90$ and $0.2 / 30 / 90$ for TTIP and $\operatorname{diH}_{2} \mathrm{O}$, respectively. The thickness and refractive index of the ALD coatings were assessed on films deposited onto a Si reference wafer (as received, $\langle 100\rangle$, native oxide layer, Si-Mat Silicon Materials) by spectroscopic ellipsometry (SENProTM, SENTECH Instruments GmbH). After ALD, the polymeric template was burned out in air in a muffle furnace at 500 ${ }^{\circ} \mathrm{C}$ for $30 \mathrm{~min}$ with a very slow heating rate of $0.3{ }^{\circ} \mathrm{C} / \mathrm{min}$.

The morphology of the photonic structure was analyzed by SEM (Zeiss Supra $55 \mathrm{VP})$. Height profiles were obtained with a $2 \mu \mathrm{m}$ radius (B-Type) stylus in a DektakXT profilometer (Bruker) under the following parameters: scan range of $65.5 \mu \mathrm{m}$, profile type "Hills", and a stylus force of $0.029 \mathrm{mN}$. A resolution of about $0.2 \mu \mathrm{m} / \mathrm{pt}$ was kept constant for all measurements by adjusting the scan length and duration. The specular and diffuse reflectance spectra between 300 $\mathrm{nm}$ and $800 \mathrm{~nm}$ were obtained using a UV/VIS spectrometer (Lambda 1050, Perkin Elmer) with Universal Reflectance Accessory (URA) at an incident angle of $8^{\circ}$ and integrating sphere accessories, respectively. The incident light beam size was selected to be $0.5 \times 0.5$ $\mathrm{cm}^{2}$ to average out any possible local signal variation and the measurements were performed in the largest homogeneous area available. Calculations of the photonic stop band (PSB) for the opals (plain base) were performed using an FCC-like structure (74\% particle packing density) formed by $200 \mathrm{~nm}$ diameter particles and refractive indexes of 1.60 and 2.12 for PS and ALD- $\mathrm{TiO}_{2}$, respectively. The effective refractive index of the PS direct opal (template) is 1.43 via the Bruggeman model, while the calculated effective refractive index of the inverse opal photonic crystal is 1.20. Since the $\mathrm{TiO}_{2}$ coating thickness is small, we simplified the surface-templated structure (after PS template burnout) to a hollow shell structure. Due to the fact that the contact points of the adjacent particles will not be coated, the volume of $\mathrm{TiO}_{2}$ is slightly overestimated in the model.

The SEM images of the particle assembly were analyzed by computer vision and machine learning techniques to investigate the similarities and differences of the particle mixes. These techniques have been developed and applied to other microstructural images. ${ }^{36}$ The image data set consists of 20 images for each mix listed in Table 1 , excluding 5 and 95 wt $\% \mathrm{NR}_{3}{ }^{+}$-functionalized PS, resulting in 220 images in total. The image analysis has two parts: 
(1) Create image representations using a pretrained deep convolutional neural network (DCNN). Previous work demonstrated that the convolution layers of DCNNs capture visual information suitable for machine learning tasks. ${ }^{37-39}$ For each SEM image in the database, a 4096-dimensional vector after the first fully connected layer $\left(f_{c} 1\right)$ of the VGG16 $\mathrm{DCNN}^{40}$ pretrained on the ImageNet data base of natural images $^{41}$ was used as the feature representation. To visualize the clustering of feature representations in $2 \mathrm{D}$, we use tdistributed Stochastic Neighbor Embedding (t-SNE) meth$\mathrm{ods}^{42}$ to embed the high-dimensional feature vectors into a $2 \mathrm{D}$ space.

(2) Perform supervised machine learning to classify the images by fine-tuning a pretrained DCNN. The particle image data set is randomly split into four subsets with 55 class-balanced images each. Three subsets (165 images) are used for training and one subset (55 images) for validation. Since the number of images in the validation set is small, a 4-fold cross-validation is performed to better assess the predictive power of the DCNN model on unseen data. For each training/validation pair, the classification layers of a pretrained ResNet50 DCNN are finetuned with the Adam optimizer and learning rate decay. ${ }^{43}$

\section{RESULTS AND DISCUSSION}

3.1. Characterization of Particle Mixes. The two particle types investigated in this study carried either trialkylammonium surface groups, denoted as $\mathrm{NR}_{3}{ }^{+}$-functionalized particles, which were positively charged, or sulfate surface groups for negatively charged particles, referred to as plain particles. PS particles are conventionally synthesized via emulsion polymerization where potassium persulfate is commonly used as a polymerization initiator compound. The persulfate initiator decomposes during the polymerization, and the resulting sulfate groups remain as surface-terminating groups on the PS particle surfaces. ${ }^{44}$ Such particles, which are not further surface-modified after particle synthesis, are thus labeled plain. However, surface functionalization with end groups such as trialkylammonium is then carried out in an additional surface modification step after the PS particle synthesis, where in this case, the PS particles react with tertiary amines, for example, trimethylamine. ${ }^{45}$ The individual zeta potential curves of plain and $\mathrm{NR}_{3}{ }^{+}$-functionalized particles are shown in Figure S1, where the $\mathrm{pH}$ range of the investigated particle mixes is indicated by the rectangular marker. Within this range from $\mathrm{pH} 2.4$ to 4.2 , plain particles and $\mathrm{NR}_{3}{ }^{+}$functionalized PS particles carry a zeta potential of approx. -35 and $+30 \mathrm{mV}$, respectively. Moreover, both particle types do not show an isoelectric point (IEP); thus, plain and $\mathrm{NR}_{3}{ }^{+}-$ functionalized particles maintain their negative and positive surface charge over the whole $\mathrm{pH}$ range analyzed. Note that the data shown in Figure S1 was provided by the particle's supplier and therefore deviate slightly from the latest results shown in Figure 2 due to the measurement parameters such as water purity and conductivity used for dilution prior to measurement. In Figure S2, the $\mathrm{pH}$ value is shown for each particle mix suspension at a different weight fraction of $\mathrm{NR}_{3}{ }^{+}$functionalized particles with plain particles as base. We chose not to adjust the $\mathrm{pH}$ of the mixes because it has been previously reported that a change in $\mathrm{pH}$ can also influence particle self-assembly and ordering. ${ }^{46,47}$

Zeta potential and agglomerate size analysis via dynamic light scattering (Figure 2) show the expected behavior of heteroaggregation between both particle types, as the amount of positively charged $\mathrm{NR}_{3}{ }^{+}$-functionalized particles added on top of the negatively charged plain particles increases. When

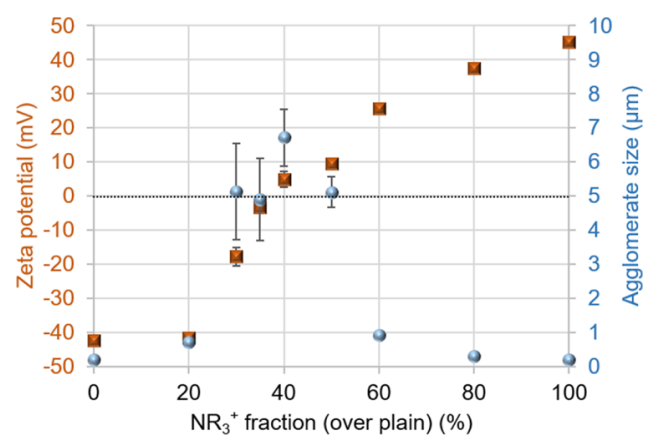

Figure 2. Variation of zeta potential and agglomerate size according to the mixing fraction of positively charged particles $\left(\mathrm{NR}_{3}{ }^{+}-\right.$ functionalized) added on top of the negatively charged particles (plain).

no $\mathrm{NR}_{3}{ }^{+}$-functionalized particles were added, the suspension containing only plain particles exhibits a zeta potential of $-42.2 \mathrm{mV}$ and an average hydrodynamic diameter of $205 \pm 2$ $\mathrm{nm}$. The addition of $\mathrm{NR}_{3}{ }^{+}$-functionalized particles to plain particles yielding a weight fraction of $20 \mathrm{wt} \%$ leads to a minor increase of the zeta potential, whereas the beginning of the agglomeration process was observed. The measured particle diameter for this mix is $725 \pm 50 \mathrm{~nm}$, which corresponds to $\sim 20$ coagulated particles in average. With further addition of $\mathrm{NR}_{3}{ }^{+}$-functionalized particles to $30 \mathrm{wt} \%$, the zeta potential of the suspension and the agglomerate sizes increase drastically. The IEP of the particle mix suspension is reached at a mixing ratio of $35 \mathrm{wt} \% \mathrm{NR}_{3}{ }^{+}$-functionalized particles, and a maximum agglomerate size of $6710 \pm 843 \mathrm{~nm}$ was observed for $40 \mathrm{wt} \%$ $\mathrm{NR}_{3}{ }^{+}$-functionalized particles at which the suspension starts showing a positive zeta potential value. Furthermore, the relatively big scattering of the agglomerate sizes for the mixes at 30,35 , and $40 \mathrm{wt} \% \mathrm{NR}_{3}{ }^{+}$-functionalized particles indicate pronounced coagulation of the oppositely charged particles. For mixes with 50 wt $\% \mathrm{NR}_{3}{ }^{+}$-functionalized particles and further, the agglomerate sizes decrease while the zeta potential is increasing until the values for the suspension containing only $\mathrm{NR}_{3}{ }^{+}$-functionalized particles are achieved. Heteroaggregation is still present for a mix with $60 \mathrm{wt} \% \mathrm{NR}_{3}{ }^{+}$-functionalized particles, forming agglomerates with a total size of $927 \pm 40$ nm. With 80 wt \% positive particles in the mix, the agglomerate size decreases down to $300 \pm 7 \mathrm{~nm}$ ( $\sim 2$ particles in average), indicating only a low level of heteroaggregation for this mix.

3.2. Film Morphology. The level of disorder of the particle assembly and the microstructure of the films fabricated from each particle mix were evaluated by analyzing the SEM images and the associated 2D fast Fourier transform (FFT) of the photonic structures shown in Figures 3 and S3. Dropcasting of a suspension with only plain base particles resulted in a typical photonic crystal film presenting an FCC-like structure for the monodisperse spherical particles with a longrange order, confirmed by the hexagonal pattern in the FFT, and a very smooth film surface (Figure 3a). Contact angle measurements of sessile droplets from the plain PS base suspension demonstrated that the substrate wetting and thus the hydrophilicity increased substantially after the plasma treatment and high wettability was still present $7 \mathrm{~h}$ after the treatment (Figure S4), that is, the maximum evaporation time. The contact angle between the wetting droplet and the substrate is related to the substrate's surface charging as the increase of charge carriers leads to decreasing contact angles. ${ }^{48}$ 


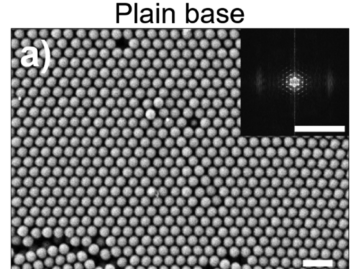

$20 \% \mathrm{NR}_{3}{ }^{+}$

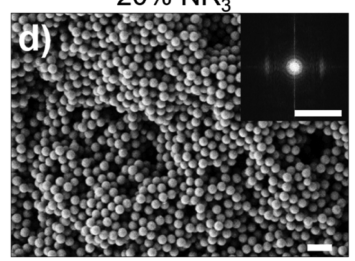

$40 \% \mathrm{NR}_{3}{ }^{+}$

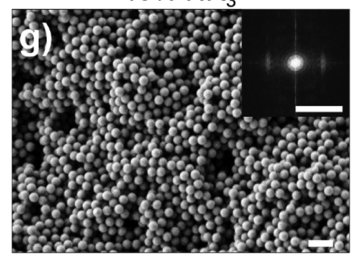

$90 \% \mathrm{NR}_{3}^{+}$

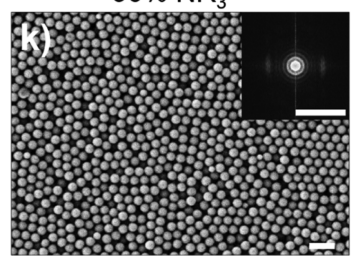

$5 \% \mathrm{NR}_{3}{ }^{+}$

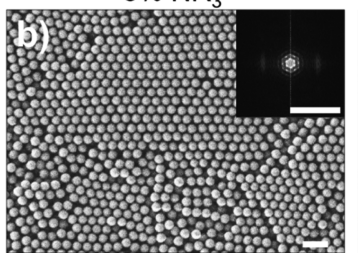

$30 \% \mathrm{NR}_{3}{ }^{+}$

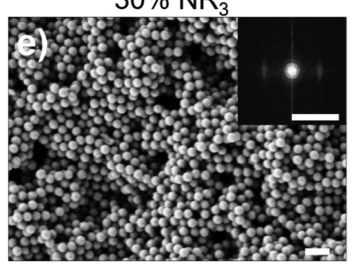

$50 \% \mathrm{NR}_{3}{ }^{+}$

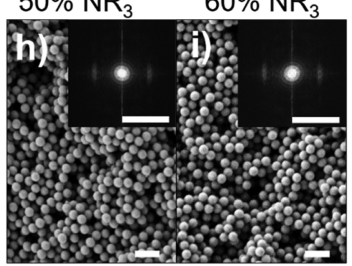

$95 \% \mathrm{NR}_{3}{ }^{+}$

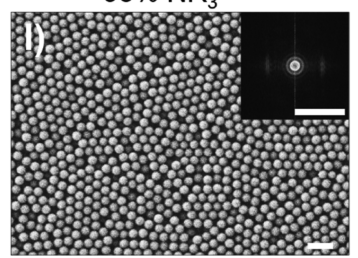

$10 \% \mathrm{NR}_{3}^{+}$

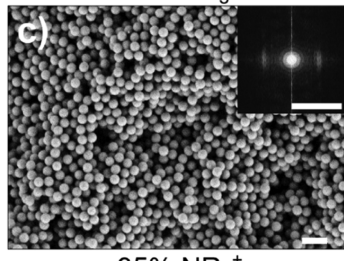

$35 \% \mathrm{NR}_{3}{ }^{+}$

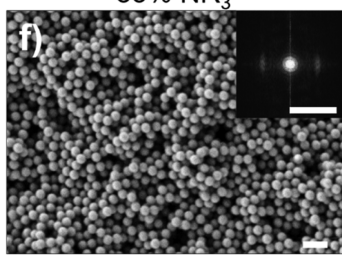

$80 \% \mathrm{NR}_{3}{ }^{+}$

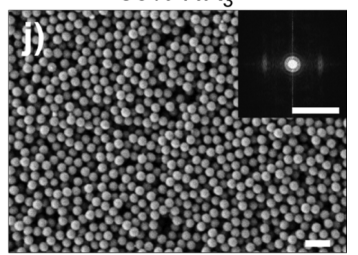

$\mathrm{NR}_{3}{ }^{+}$base

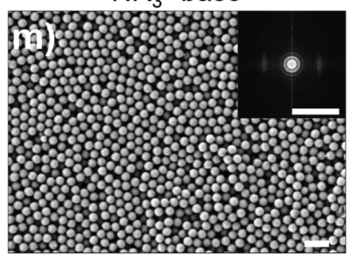

Figure 3. $(\mathrm{a}-\mathrm{m})$ Top view SEM images and 2D fast Fourier transform of the photonic structures according to the different base particles and different mixes (see Table 1 for details). An enlarged version of the FFT images is shown in Figure S3. Scale bars represent $500 \mathrm{~nm}$ for the SEM images and $0.4 \mathrm{~nm}^{-1}$ for the FFTs. Particle fractions correspond to weight percent (see Table 1 for details).
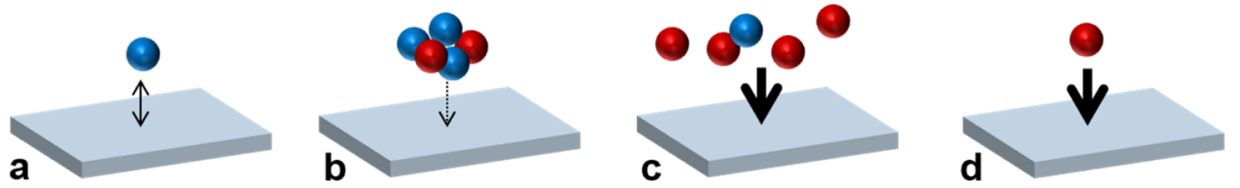

Figure 4. Schematic drawing of different interaction scenarios between particles and the substrate for different mixing ratios. Blue spheres represent negatively charged particles and red spheres represent positively charged particles. The substrate carries a negative surface charge in all scenarios. Solid arrows indicate the electrostatic interaction in (a) repulsive and (c) and (d) attractive forces, while the dotted arrow in (b) denotes the sedimentation process.

Moreover, the number of oxygen atoms bound to the substrate surface increases after plasma treatment ${ }^{49}$ and the higher number of oxygen (number of ionizable sites) is related to a more negative zeta potential. ${ }^{50}$ Thus, the plasma-treated sapphire substrates used in this work can be considered as negatively charged.

In this case, the highly ordered, crystalline structure was obtained by evaporation-induced self-assembly ${ }^{51}$ where electrostatic repulsive forces act between the negatively charged plain particles themselves and the negative surface of the plasma-treated sapphire substrate as indicated in Figure 4a. Hence, the particles have high mobility on the substrate to finally arrange in the energetically favorable lattice. The introduction of $5 \mathrm{wt} \%$ positively charged $\mathrm{NR}_{3}{ }^{+}$-functionalized particles to the plain particles still results in a crystalline structure (Figure $3 \mathrm{~b}$ ). However, the FFT shows less distinctive spots than before, which reveals that the long-range order is disturbed already with the little amount of positively charged particles, and hence, the ordered domain size is reduced in comparison to the plain base sample.

The transition from an ordered particle deposition to disorder occurs from 5 to $10 \mathrm{wt} \% \mathrm{NR}_{3}{ }^{+}$-functionalized particles; that is, at least $10 \mathrm{wt} \%$ of positively charged particles are necessary to suppress the self-assembly process on due to the heteroaggregation of oppositely charged particles. Due to the attractive electrostatic forces between plain particles and $\mathrm{NR}_{3}{ }^{+}$-functionalized particles, micrometer-sized agglomerates are formed in the aqueous suspension, which subsequently sedimented onto the substrate (Figure $4 \mathrm{~b}$ ). This process leaves behind loosely packed, disordered particle deposits with a short-range order, revealed by concentric circles in the FFT, with a rough film surface as also observed for the mixes from 10 to 60 wt $\% \mathrm{NR}_{3}{ }^{+}$-functionalized particles (Figure $3 \mathrm{c}-\mathrm{i}$ ). The rough film morphology agrees with the observations from the zeta potential and agglomerate size measurements of the suspensions in Figure 2. The observation of higher porosity in the films dominated by heteroaggregation is also supported by 


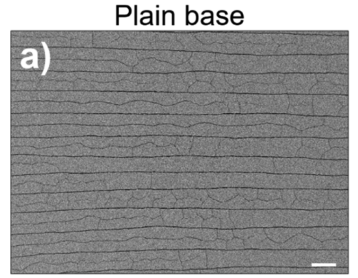

$20 \% \mathrm{NR}_{3}^{+}$

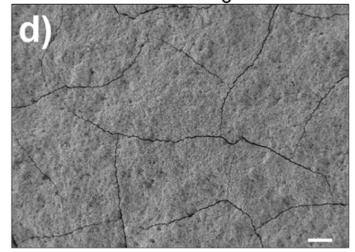

$40 \% \mathrm{NR}_{3}{ }^{+}$

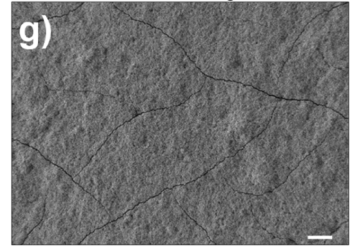

$90 \% \mathrm{NR}_{3}{ }^{+}$

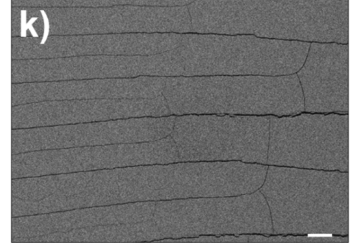

$5 \% \mathrm{NR}_{3}{ }^{+}$

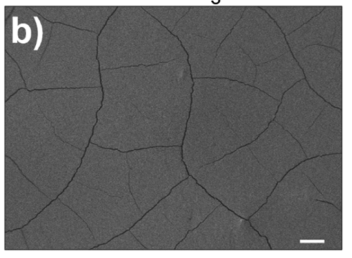

$30 \% \mathrm{NR}_{3}{ }^{+}$

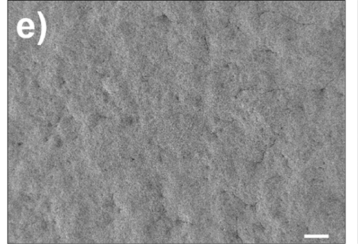

$50 \% \mathrm{NR}_{3}^{+} \quad 60 \% \mathrm{NR}_{3}{ }^{+}$

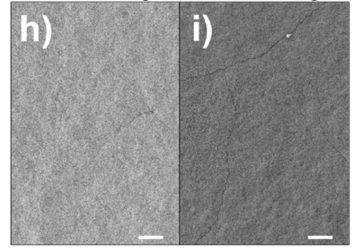

$95 \% \mathrm{NR}_{3}{ }^{+}$

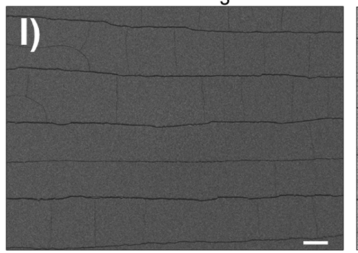

$10 \% \mathrm{NR}_{3}{ }^{+}$

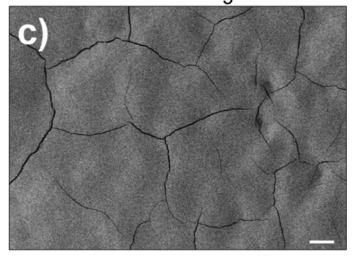

$35 \% \mathrm{NR}_{3}{ }^{+}$

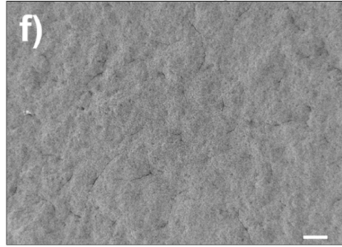

$80 \% \mathrm{NR}_{3}{ }^{+}$

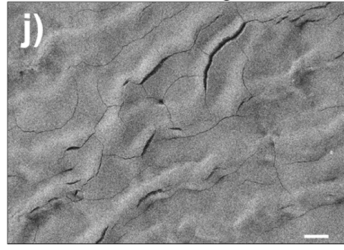

$\mathrm{NR}_{3}{ }^{+}$base

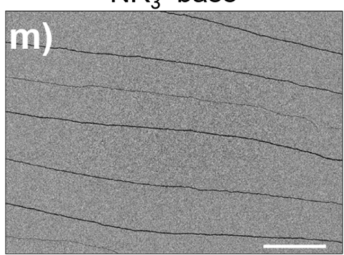

Figure 5. $(\mathrm{a}-\mathrm{m})$ Top view low-magnification SEM images of the photonic structures according to the different base particles and different mixes (see Table 1 for details). Scale bars represent $100 \mu \mathrm{m}$. Particle fractions correspond to weight percent (see Table 1 for details).

the cross-sectional SEM images of particle mixes with 10 and 50 wt $\% \mathrm{NR}_{3}{ }^{+}$-functionalized particles (Figure S5a,b).

From 60 to 80 wt $\% \mathrm{NR}_{3}{ }^{+}$-functionalized particles, we note another microstructural transition from a disordered particle arrangement with high porosity and rough surface to a still disordered deposit but with higher packing density and smoother surface. Figure $3 \mathrm{j}$ shows the SEM image of the film deposited from the mix with $80 \mathrm{wt} \% \mathrm{NR}_{3}{ }^{+}$-functionalized particles. In this case, the results from agglomerate size measurement showed a smaller degree of heteroaggregation with agglomerate size in the order of $300 \mathrm{~nm}$ for $80 \mathrm{wt} \%$ versus $927 \mathrm{~nm}$ for $60 \mathrm{wt} \%$. Notably, the morphology of the 80 wt $\% \mathrm{NR}_{3}{ }^{+}$mix differs from the 20 wt $\% \mathrm{NR}_{3}{ }^{+} \mathrm{mix}$, although the ratio between positively and negatively charged particles is the same. This is related to the different agglomerate sizes, 725 $\mathrm{nm}$ for $20 \mathrm{wt} \% \mathrm{NR}_{3}{ }^{+}$-functionalized particles, and the sapphire substrate charge, which is negative after the oxygen plasma treatment. Therefore, since the global zeta potential for particles in this mix ( $80 \mathrm{wt} \% \mathrm{NR}_{3}{ }^{+}$-functionalized particles) was positive, it led to an attractive electrostatic force between particles and the substrate surface, inhibiting free lateral movement of the particles on the substrate surface, thus preventing packing with a crystalline order rather than only sedimentation as in the case of the $20 \mathrm{wt} \% \mathrm{mix}$, as illustrated schematically in Figure 4c. For 90 and 95 wt $\% \mathrm{NR}_{3}{ }^{+}$ functionalized particles, the particles in the film are more closely packed and the volume of the interstitial pores decreases, as shown in the cross-sectional SEM image in Figure S5c, and small ordered islands with size $\geq 1 \mu \mathrm{m}$ occur (Figure 3k). Moreover, distinct short-range anisotropy was observed for the particle mixes with 5, 90 and $95 \mathrm{wt} \% \mathrm{NR}_{3}{ }^{+}$ functionalized particles. This was not observed for the other particle mixes due to the fact that those were dominantly affected by the heteroaggregation of the oppositely charged particles. Thus, free particle movement and particle rearrangement creating short-range anisotropy were strongly suppressed by the particles' attractive electrostatic interaction.

The film from $\mathrm{NR}_{3}{ }^{+}$-functionalized base particles resulted in a random closely packed structure, which shows more rings in the FFT compared to the other disordered arrangements, indicating a higher number of correlated particles. ${ }^{52}$ The particle-substrate interaction in this particular case is strongly attractive since the particles and the substrate carry opposite surface charges (Figure 4d). Again, the particles close to the substrate surface are immobilized and consequently deposit in a disordered structure, corroborated by the results obtained by Yan et al. ${ }^{53}$ On the contrary, the repulsion between the positive $\mathrm{NR}_{3}{ }^{+}$-functionalized particles may lead to small ordered areas especially in the film top layer, which do not interact with the substrate.

The different particle-particle and particle-substrate interactions shown in Figure 4 have a significant effect on the resulting film stability regarding crack formation. The evaluation of film defects such as cracks in self-assembled crystals and glasses is important since cracks substantially introduce light scatterings and will lower the photonic performance of the film. ${ }^{54}$ In Figure 5, low-magnification SEM images of each film with different particle mix compositions illustrate the crack morphology. The film with only plain base particles experienced severe cracking during the 
drying process. The observed linear cracks, which result from capillary forces and tensile stresses due to the evaporation of interstitial water between the particles, are typical for highly ordered colloidal assemblies. ${ }^{55-58}$ Alternatively, films fabricated from suspension mixes with 5, 10, 20, and 40 wt \% show an irregular crack pattern. We hypothesize that this could be related to the combination of assembly via heteroaggregation and sedimentation with evaporative self-assembly. The particle mixes that assembled into disordered films with a rough surface, discussed in the previous paragraphs, also present a rough surface appearance in lower magnification.

Films of the mixes with 30, 35, 50, and 60 wt \% exhibit minor crack formation. In these cases, the evaporation rate during the film drying process was significantly faster than for the base particles (Figure 6). Generally, slower water

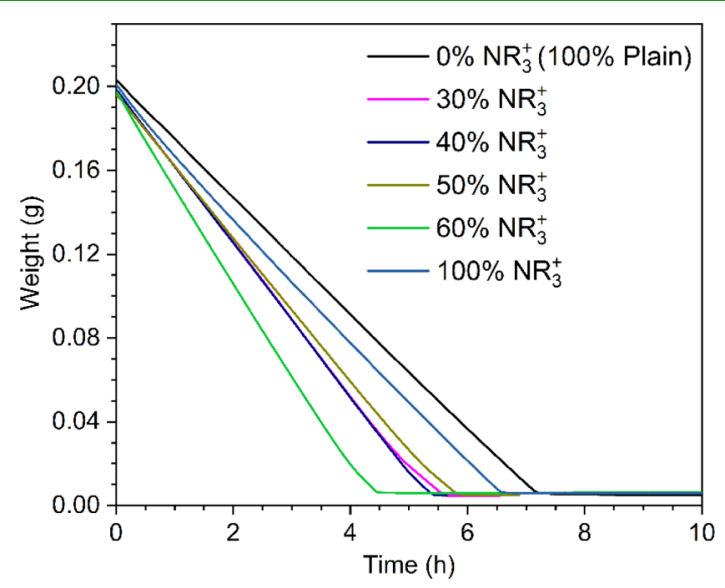

Figure 6. Weight loss over time during film drying for selected mixes. The number of plots shown in this figure was reduced for clarity. All the plots are shown in Figure S6. Particle fractions correspond to weight percent (see Table 1 for details).

evaporation generates less cracks compared to faster evaporation rates. ${ }^{59}$ Here, the fast evaporation is related to the suggested deposition process in Figure $4 \mathrm{~b}$, which is governed by the sedimentation of agglomerated particles. Due to the resulting loose packing and high porosity, less interstitial water is trapped between the agglomerates. As a result of that, the films dry faster and the capillary forces that usually cause crack formation are globally reduced. Therefore, less cracks are observed in these cases. Nonetheless, there is a limit of agglomerate size for which the film assembles with minor crack formation. For instance, the film formed by the $40 \mathrm{wt} \% \mathrm{NR}_{3}^{+}$ mix particles showed the presence of several cracks. This particles' mix presented the largest average agglomerate size of $6.7 \mu \mathrm{m}$, which is $30 \%$ bigger than both 30 and $50 \mathrm{wt} \% \mathrm{NR}_{3}^{+}$ mixes. In case such agglomerates were to be considered as spheres, this would mean a substantial average increase of $\sim 90 \%$ in the sedimentation rate of such particles (calculated via Stokes' Law). Although the agglomerates are most likely not perfectly spherical, one can assume that the sedimentation rate of $\sim 7 \mu \mathrm{m}$ will be way higher than $\sim 5 \mu \mathrm{m}$ agglomerates. This would lead to agglomerate sedimentation following the case of Figure $4 \mathrm{~b}$ for both cases, but while in the 30 and $50 \mathrm{wt}$ $\% \mathrm{NR}_{3}{ }^{+}$mixes, the agglomerates possibly still have time for rearrangement prior to solvent evaporation but not in the 40 wt $\% \mathrm{NR}_{3}{ }^{+}$mix.

The height profiles displayed in Figure 7 support the SEM analysis of the films' morphology. Films that presented a

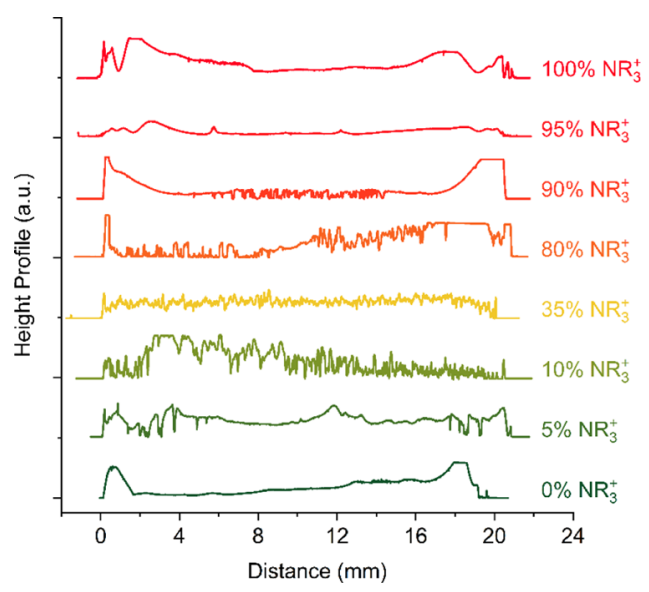

Figure 7. Height profiles of a selection of as-fabricated films with different amounts in weight percent of $\mathrm{NR}_{3}{ }^{+}$-functionalized particles in the mix. All profiles are shown in Figure S7.

smooth surface in the SEM images show smaller height variations in the height profile curve as observed for $0,5,90$, 95, and $100 \mathrm{wt} \% \mathrm{NR}_{3}{ }^{+}$-functionalized particles when in comparison to rougher structures represented by samples with 10 to 80 wt $\% \mathrm{NR}_{3}{ }^{+}$-functionalized particles which presented strong height fluctuations (height profiles of all samples are plotted in Figure S7). This roughness influences the overall color impression, with rougher films presenting a matte appearance (discussed in detail in the "Optical Properties" section). These films present, however, a suppression of the coffee-ring effect due to the fast sedimentation of agglomerated particles, which causes a reduction of the convective particle flux toward the edges of the drop-casted suspension droplet. While films with $20-60 \mathrm{wt} \% \mathrm{NR}_{3}{ }^{+}$-functionalized particles exhibit a homogeneous or dome-like film height profile, where no coffee ring was observed, films of the base particle mixes, namely, 0 and 100 wt $\% \mathrm{NR}_{3}{ }^{+}$-functionalized particles, presented a very pronounced coffee-ring effect. Note that the PhG films fabricated with 10 and 80 wt $\% \mathrm{NR}_{3}{ }^{+}$-functionalized particles represent the transition from smooth to rough surface and vice versa, displaying in both cases quite inhomogeneous and asymmetric film heights over the entire profile scan. Concerning the film thickness, films with higher porosity have a lower packing density, which results in a comparatively higher average film thickness $(\sim 37 \mu \mathrm{m})$, while the total particle amount was kept constant in all mixes. As the particle packing becomes denser for 80 up to $100 \mathrm{wt} \% \mathrm{NR}_{3}{ }^{+}$-functionalized particles in the mix, the height of the films decreases considerably to $\sim 20 \mu \mathrm{m}$. As demonstrated in a previous study, films with lower thickness present an enhanced structural color effect due to reduced multiple scattering events within the film. ${ }^{60}$ Moreover, a recent review has shown that improved structural coloration can be achieved with higher packing densities. ${ }^{5}$ Both characteristics are tailored and improved by the developed heteroaggregation-based process.

3.3. DCNN Image Analysis. The goal of the DCNN image analysis is the ability to reveal information that cannot be revealed by conventional means for capturing and understanding the surface charge-mediated structural transitions in photonic glasses. Visual cluster analysis reveals similarities and differences between images that correspond to different types of assembly structures. An image classification challenge elucidates whether structures that appear similar contain 
subtle, but systematic differences, which could not be detected by conventional methods.

The foundational task of computer vision is defining a feature vector, which is a mathematical representation of the visual information contained in an image. For microstructural images, feature vectors obtained from convolution layers of pretrained DCNNs have proved to capture rich information at different length scales. ${ }^{37-39}$ Although DCNNs are typically pretrained on natural images (e.g., photographs of cars, cats, carnations, and so forth), and not on photonic glass images, they learn many low-level details like edges, blobs, and visual textures that apply quite generally to microstructures and natural images alike. For photonic glass images, the pretrained DCNN can capture particle boundaries, shapes, arrangements, and relative positions and can encode this information into the feature vector.

To visualize the feature vectors for the photonic glass images, Figure 8 shows a t-SNE plot, where each point

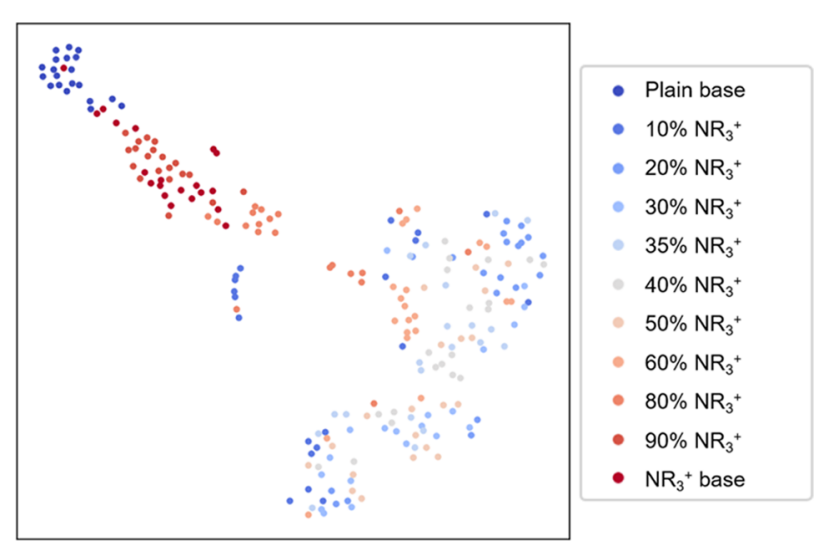

Figure 8. t-SNE plot of DCNN feature representations of the SEM images. Each point represents the $2 \mathrm{D}$ projection of the feature vector of an SEM image, and the distance between points scales with visual similarity between images; nearby points resemble each other visually. Data point color corresponds to the $\mathrm{NR}_{3}{ }^{+}$-functionalized particle fraction in weight percent, as shown in the legend.

represents an image, and the distance between each pair of points is related to the visual similarity between the two images (note that the high-dimensional space of the feature vectors is reduced to 2-D for the t-SNE plot, so some information is lost. In particular, the distance between points in the 2-D plot is nonlinearly related to their high-dimensional distance). In Figure 8, the color of the data points changes from blue to red with the increasing fraction of $\mathrm{NR}_{3}{ }^{+}$-functionalized particles in the particle mixes. The plain base particles (dark blue) have an FCC-like structure with a long-range order, and the data points are closely grouped together at the top left corner. As the $\mathrm{NR}_{3}{ }^{+}$-functionalized particle fraction increases to $10 \mathrm{wt} \%$, the particles transition to a partially disordered structure, and the data points (medium blue) cluster in the center of the plot. The data points of particle mixes with 20 to $60 \mathrm{wt} \% \mathrm{NR}_{3}{ }^{+}$functionalized particles intermingle in a loose cluster in the bottom right region. These images are hard to distinguish from each other because all of these particle mixes display a high level of disorder and porosity. When the $\mathrm{NR}_{3}{ }^{+}$-functionalized particle fraction rises to $80 \mathrm{wt} \%$, the data points (medium red) shift back to the center as a result of the transition of the particles from a loosely packed structure to a densely packed structure. For 90 wt $\% \mathrm{NR}_{3}{ }^{+}$mixes and $\mathrm{NR}_{3}{ }^{+}$-functionalized base particles, the data points (dark red) locate near the plain base particles since they have a randomly closely packed structure with patches of ordered regions that is visually more similar to the crystalline structure (see Figure 3 ). The plot explicitly shows the transitions from a highly ordered structure to a disordered structure and back to an ordered structure, capturing the same phenomenology exhibited in the spectroscopic measurements (discussed in detail in the "Optical Properties" section). This is the first demonstration and validation of DCNN-based computer vision as a structural analysis method for particulate materials.

A question that is difficult to answer using spectroscopic measurements is whether the disordered structure observed for 20-80 wt $\% \mathrm{NR}_{3}{ }^{+}$-functionalized particles is similar or whether it varies with $\mathrm{NR}_{3}{ }^{+}$-functionalized particles fraction. To find evidence for subtle structural differences between particle mixes, the ResNet50 DCNN (Figure S8) was fine-tuned and trained to perform supervised classification of the 11 classes of images. For this task, the DCNN achieves an accuracy score at $88.6 \pm 2.7 \%$, demonstrating that it can differentiate particle mixes according to $\mathrm{NR}_{3}^{+}$fraction.

To evaluate the model predictions in a systematic way, a confusion matrix is constructed to show the performance of predictions by class, as shown in Figure 9. The score along the

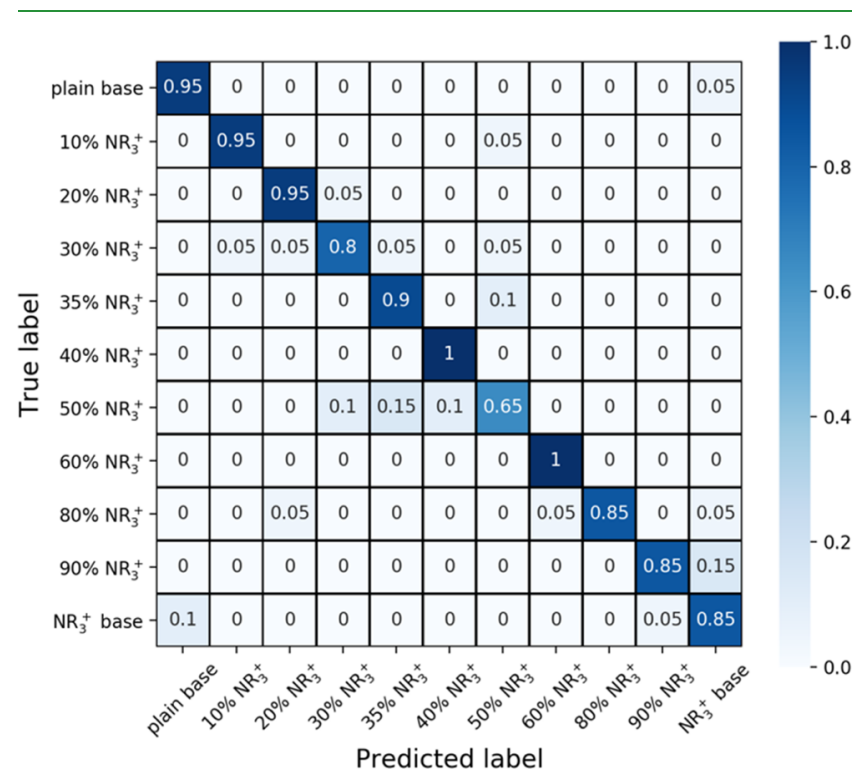

Figure 9. Confusion matrix for the DCNN classification results with a 4-fold cross-validation. The vertical axis shows the true class label of an image and the horizontal axis gives the predicted label. The value in the cell indicates the proportion of the images from row label that are predicted to have the column label.

diagonal represents the proportion of the images for which the true label and the predicted label are the same, which is a good way to measure the performance for each particle mix. The classification scores for plain base and $10 \mathrm{wt} \% \mathrm{NR}_{3}{ }^{+}$ functionalized particles are high, consistent with the t-SNE plot, which indicates that they form distinct visual clusters of images. More surprisingly, the $20 \mathrm{wt} \% \mathrm{NR}_{3}{ }^{+}$-functionalized particle sample also has high classification accuracy, indicating that it contains visually distinctive features (note that the method unfortunately does not reveal what those features are). Likewise, the random closely packed structures of $90 \mathrm{wt} \%$ $\mathrm{NR}_{3}{ }^{+}$and $\mathrm{NR}_{3}{ }^{+}$-functionalized particle base are classified with 
high accuracy, but because they are very similar, there is a certain degree of confusion between these two classes. The $\mathrm{NR}_{3}{ }^{+}$-functionalized particle base images can also be mistakenly identified as the plain base because of the patches of crystalline order in between the randomly packed disorder structure.

The intermediate mixes, which did not show significant clustering in the $\mathrm{t}$-SNE plot, nonetheless achieve the overall high classification scores, indicating that they are visually different from one another. Accuracy for mixes between 30 and 60 wt $\% \mathrm{NR}_{3}{ }^{+}$-functionalized particles varies without obvious trend, with 40 and 60 wt \% being the best and 50 wt \% being the worst. Images from $50 \mathrm{wt} \% \mathrm{NR}_{3}{ }^{+}$-functionalized particles are sometimes predicted as 30,35, and $40 \mathrm{wt} \%$ but never 60 wt $\%$. All mixes are classified with an accuracy that is much higher than the random guessing score of 0.09. Thus, even though particle images with $\mathrm{NR}_{3}{ }^{+}$-functionalized particles fraction from 20 to $60 \mathrm{wt} \%$ appear very similar, and their images intermingle on the t-SNE plot, the DCNN computer vision system is able to tell the images of the different particle mixes apart. Interpretation of the DCNN model is important but very challenging due to the high complexity of the models and the implicit way the image information is represented. We hypothesize that the DCNN model can detect high-level features of the images like the particle agglomerate size and the porosity size, which can be helpful in differentiating highly disordered particle images from different mixes.

Overall, supervised training of the DCNN model can achieve high classification performance for multiple types of particle mixes. Although images from certain classes cannot be visually distinguished by humans or greatly differentiated by the spectroscopy analysis (refer to the next section), there is some hidden information that can be detected by the DCNN model and used for making distinctions. Moreover, once the training is done, classification is quite straightforward, and selection or elimination of structures that do or do not correspond to the desired structure to generate structural colors can be performed within hours. The major drawback of the DCNN model is that the information it detected is hard to interpret, and we cannot directly connect it to a quantitative evaluation of particles arrangement. It is worth exploring and demystifying the information in the future to come up with a solution to correlate images directly with structures and properties.

3.4. Optical Properties. The optical properties of colloidal assemblies depend mainly on particle ordering, particle packing, and the difference between the refractive indexes of the particles and the matrix. Here, the as-fabricated films vary in their degree of order and packing, as demonstrated in Figure 3 and subsequent discussion. In the specular reflection spectrum of the film from plain base particles (Figure 10), a distinctive peak is located at $455 \mathrm{~nm}$. This peak is connected to the highly ordered particle assembly and represents the characteristic photonic band gap of photonic crystals. ${ }^{51,61}$ As the amount of $\mathrm{NR}_{3}{ }^{+}$-functionalized particles in the mix increases, the resulting structures exhibit a higher level of disorder with respect to plain base films. Therefore, the reflectance peak at $455 \mathrm{~nm}$ decreases notably already with $5 \mathrm{wt}$ $\% \mathrm{NR}_{3}{ }^{+}$-functionalized particles and vanishes completely with 10 wt $\% \mathrm{NR}_{3}{ }^{+}$-functionalized particles in the mix where the structure shows a complete disorder on the microscale (see Figures 3 and S5), plus the film exhibits a rough surface. Films with $20-80$ wt $\%$ positive particles show similar reflection spectra for specular reflectance and no peak is observed

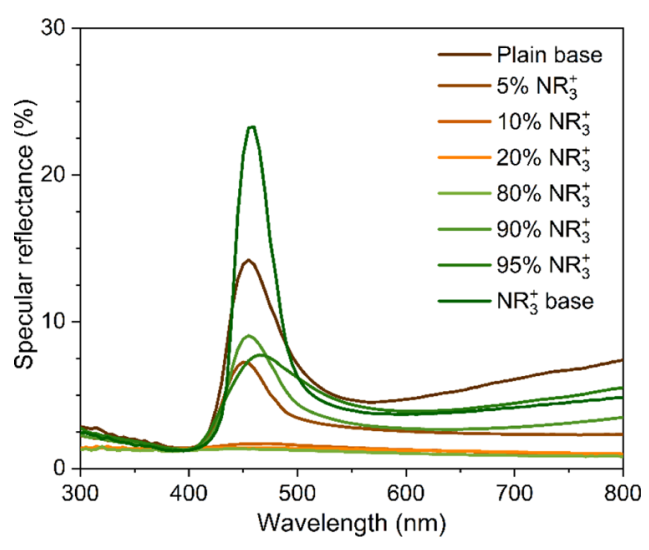

Figure 10. Specular reflection spectra of the photonic structures according to the different base particles and different mixes. Particle fractions correspond to weight percent (see Table 1 for details). The number of spectra shown in this figure was reduced for clarity. All the spectra are shown in Figure S9.

(Figure S9). Interestingly, a peak appears again in the spectra of the films from 90,95 , and 100 wt $\% \mathrm{NR}_{3}{ }^{+}$-functionalized particle mixes with increasing reflectance, even though the particles still present a disordered arrangement. In these cases, the specular reflectance peak may be attributed to the smooth surface of the films and the small ordered areas observed on the upper layer, while the bulk of the film is disordered, as visualized in Figure S10. A similar observation was reported by Chen et al., ${ }^{15}$ where the authors combine a disordered particle assembly with a smooth and ordered upper layer to optimize the optical appearance of structural color films from matte into shiny. Note that in this work, the disordered particle arrangement was induced by heteroaggregation of particles with the same diameter and therefore no shift of the reflectance peak was observed, whereas for bimodal particle films, the peak shifts significantly depending on the concentration of the second particle species of different size. ${ }^{11}$

The diffuse reflectance over the visible wavelength spectrum (Figures 11 and S11) increases with the addition of positive particles reaching above $90 \%$ reflectance for the mix with $20 \mathrm{wt}$ $\% \mathrm{NR}_{3}{ }^{+}$-functionalized particles. Films with lower or higher fraction of positive particles show a less diffuse reflectance,

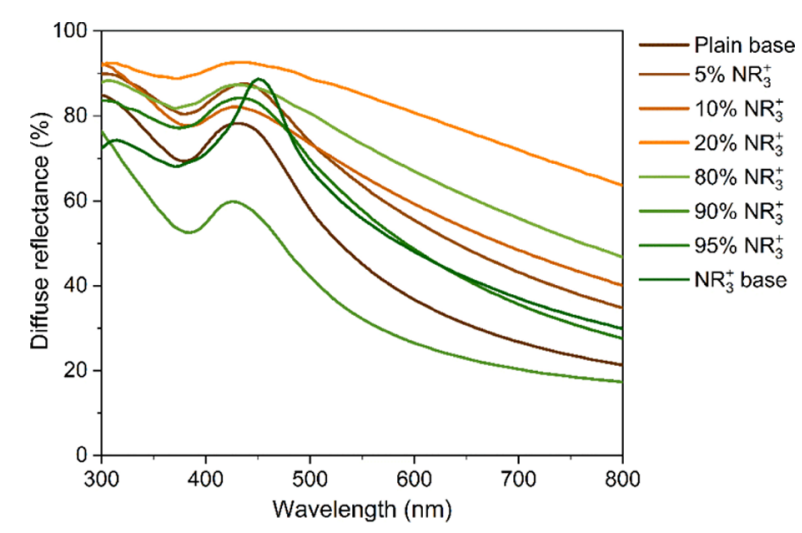

Figure 11. Photonic structures diffuse reflection spectra according to the different base particles and different mixes. Particle fractions correspond to weight percent (see Table 1 for details). The number of spectra shown in this figure was reduced for clarity. All the spectra are shown in Figure S11. 
especially in the long-wavelength range. This observed increase of diffuse reflection behavior is connected to the disordered particle deposition and rough surface characteristics of the films which are driven by the heteroaggregation process developed in this work.

The motivation to tailor the ordering and packing of the photonic glass films presented in this work was to optimize the templates for the generation of inverted structures. This is achieved by infiltrating the structures via ALD and removing the template, generating structures with high porosity, low density, and high surface-to-volume ratio. ${ }^{29,30,62}$ Optical simulations for an inverted disordered assembly revealed that coating of the deposited $200 \mathrm{~nm}$ PS particles with a $\mathrm{TiO}_{2}$ layer of $10 \mathrm{~nm}$ shell thickness via ALD would yield a structure with optimized reflectance regarding structural coloration. The theoretical background and optical simulations of surfacetemplated inverse photonic glasses are demonstrated in our recent work. ${ }^{35}$

Titania was chosen as a shell material because of its relatively high refractive index. Furthermore, such ceramic-based photonic structures can be used for high-temperature applications due to the high structural stability up to 1000 ${ }^{\circ} \mathrm{C}$ for titania explored in a former publication from our group. ${ }^{65}$ The packing density and degree of disorder affect the photonic performance as discussed above. Therefore, we selected photonic structures that show different packing and film morphologies based on both the SEM images and the DCNN analysis, namely, 0 wt \% (plain base), 35, 50, 95, and 100 wt $\% \mathrm{NR}_{3}{ }^{+}$-functionalized particles.

After ALD coating with $12 \mathrm{~nm}$ of $\mathrm{TiO}_{2}$ and subsequent removal of the PS template via calcination, surface-templated inverse photonic glasses were obtained. Hence, the reflectance in the short visible wavelength range below $350 \mathrm{~nm}$ as well as in the longer wavelength range above $500 \mathrm{~nm}$ is substantially reduced, as demonstrated in the diffuse reflection spectra of Figure 12. Optical simulations of such surface-templated

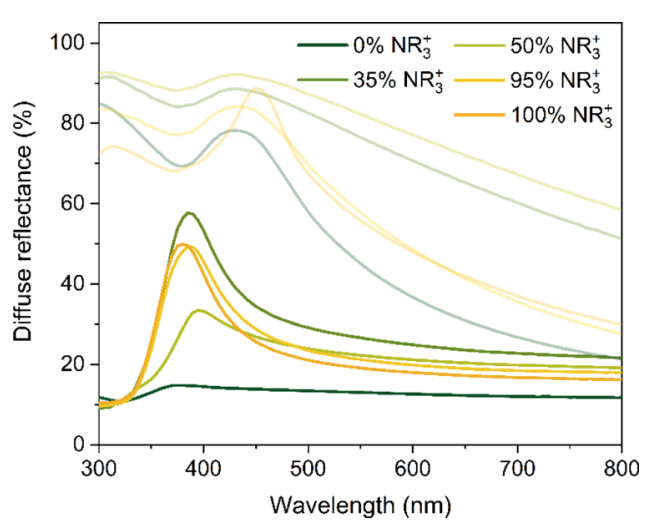

Figure 12. Diffuse reflection spectra of inverse photonic structures after ALD coating with $12 \mathrm{~nm}$ titania and subsequent calcination to remove the PS template (saturated colored lines). Faded color lines represent the diffuse reflection spectra of the photonic glass PS template (before ALD and calcination).

inverse photonic glass with thin coating thickness revealed that the inverse structure reduces mainly longer wavelength reflection while increasing the reflectance at short wavelengths. ${ }^{37}$ Thus, the suppressed reflection in the shortwavelength range observed here is due to the characteristic band gap of $\mathrm{TiO}_{2}$, which leads to a strong absorption below $\sim 390 \mathrm{~nm} .{ }^{63}$ The calculated PSB of the $200 \mathrm{~nm}$ PS direct opal (template, Figure 11-plain base) is expected at $467 \mathrm{~nm}$ for (111) direction from Bragg's law, while the calculated PSB position is expected at $392 \mathrm{~nm}$ for the inverted structures (Figure 12). The minor mismatch of the calculated positions with the measured positions is associated with the particle size variation and the approximation of the effective media theory. ${ }^{61}$ The remaining reflectance peak after inversion compared to the broad reflection from the PS template generates an improved structural color impression, which can be observed in the optical pictures of the PS templates and inverse photonic glasses in Figure 13. Note that the pictures

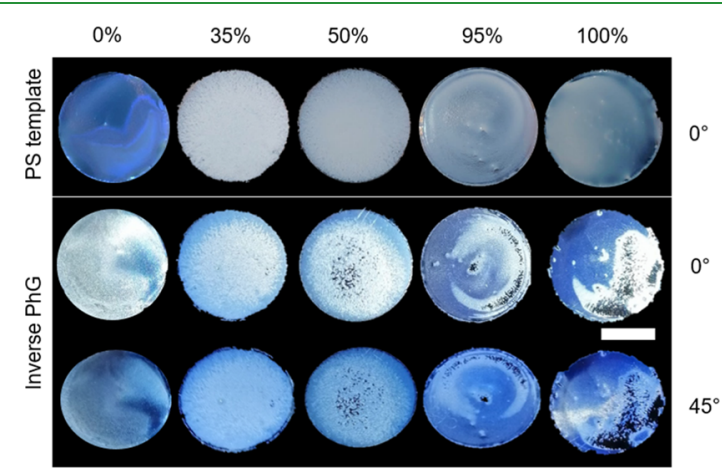

Figure 13. Optical pictures of photonic structures samples with different mixing fractions of $\mathrm{NR}_{3}{ }^{+}$-functionalized particles $(0,35,50$, 95, and $100 \mathrm{wt} \%)$ before and after ALD coating and subsequent removal of PS template spheres. The viewing angle for the PS template films is $0^{\circ}$. The inverse photonic glass films are shown under 0 and $45^{\circ}$ viewing angles. The scale bar represents $1 \mathrm{~cm}$.

were taken placing the films on a black background. ${ }^{64}$ Due to the disordered particle arrangement, the obtained structural coloration is non-iridescent as demonstrated by the optical pictures taken at $45^{\circ}$ viewing angle. Although 95 and 100 wt \% $\mathrm{NR}_{3}{ }^{+}$-functionalized PS template films showed already a bluish color, the inversion leads to an enhanced saturated blue structural color due to the suppression of reflectance in longer wavelengths. Note that the blue color impression of the plain base ( 0 wt $\% \mathrm{NR}_{3}{ }^{+}$-functionalized particles) PS template film originates from the particles' assembly into a photonic crystal and thus the structural color is strongly angle-dependent. The inversion of the plain base film resulted in a milky transparent appearance, which is revealed by the overall low reflectance of this film shown in Figure 12. The inverse photonic glass made from a loosely packed 35 wt $\% \mathrm{NR}_{3}{ }^{+}$-functionalized particle film shows the highest intensity peak in the diffuse reflection spectrum but also the reflection of longer wavelengths is stronger. Hence, the inverted film exhibits less saturated blue structural color compared to the inverted photonic glasses from closely packed 95 and 100 wt \% positive particle films. These findings point out that not only the degree of ordering of the particles but also the packing of the particles are key parameters in the process of designing and fabricating optimized structural colors.

Besides the color appearance, the film stability regarding crack formation and delamination of the final inverted films is an important aspect for the evaluation of high-quality structural colored ceramic films. The inverse PhG film from 35 wt \% $\mathrm{NR}_{3}{ }^{+}$-functionalized particles is the only film in this study, which shows blue structural coloring, for which neither cracking nor delamination of the film was observed on the 
macroscale (Figure 13). Furthermore, drop-casting of a suspension with $35 \mathrm{wt} \%$ positive particles resulted in a very homogeneous PS template film over the complete sample area of $3.14 \mathrm{~cm}^{2}$ with the successful suppression of the coffee-ring effect, as shown in the optical pictures and the height profile (Figure 7). This is particularly interesting for further developments targeting large-scale fabrication processes or 3D printing of PS-templated ceramic structural colors. Consequently, the best results considering the reflection behavior, structural color impression, and film stability was obtained for the inverted structure from $35 \mathrm{wt} \%$ positive particles with the highest reflectance of $57 \%$ at $385 \mathrm{~nm}$. In conclusion, the optimum PS template for an inverse photonic glass functioning as blue structural color was achieved by dropcasting a suspension with 35 wt $\% \mathrm{NR}_{3}{ }^{+}$-functionalized particles over plain particles. The achieved blue structural coloration is comparable to the results from Forster et al., ${ }^{13}$ where PS spheres of two different sizes were used to obtain a disordered assembly. In our approach, the structural color is generated by a ceramic-based surface-templated inverse photonic glass of titania, which can withstand temperatures of up to $1000{ }^{\circ} \mathrm{C}$ in air atmosphere. ${ }^{65}$ Meanwhile, PS-based structures soften at about $100{ }^{\circ} \mathrm{C}$ and decomposition starts at about $300{ }^{\circ} \mathrm{C}$ in air. ${ }^{66}$ Conversely, the inverse photonic glasses produced here can be used in a wider range of application temperatures than other polymeric-based conventional structural colors. Moreover, by ALD, it is possible to infiltrate the heteroaggregated templates with high-temperature materials, for example, mullite. ${ }^{30}$ Note, however, that the ALD thickness would have to be adjusted taking into account the material refractive index and the particle size used for heteroaggregation. $^{35}$

\section{CONCLUSIONS}

A heteroaggregation-based process was demonstrated to fabricate photonic glass films with tailored disorder and optimized homogeneity. Films with different microstructures transitioning from crystalline to loosely and random closely packed were obtained by varying the ratio between negatively charged and positively charged PS particles in the suspension mixes. The diversity of the resulting microstructures was related to the interaction between particles and the substrate as well as the particle-particle interaction and agglomerate formation. The heteroaggregation of equal-sized oppositely charged particles also affected the film quality and homogeneity, namely, film roughness, film thickness, crack formation, and coffee-ring suppression. The as-prepared photonic glass film fabricated from the suspension mix with $35 \mathrm{wt} \% \mathrm{NR}_{3}{ }^{+}$ functionalized particles presented no cracks and homogeneous film coverage over the entire sample area, that is, the coffeering formation was inhibited.

The optical properties showed that the crystalline microstructures presented typical optical characteristics of photonic crystals, whereas these features were decreased and eventually vanished, as the degree of disorder was increased. Furthermore, DCNN was introduced as a tool to classify and distinguish particle assembly structures based on SEM images. DCNN analysis showed that it is possible to make distinctions among particle assembly with different mixing fractions, although they have similar visual appearances. This indicates that DCNN would be a promising tool to assist the characterization of particle arrangements simply from images. However, disordered structures were sometimes misclassified. Here, an outlook could be to use additional data (e.g., profilometry data and reflection spectra) to add more features (like film thickness), which may be useful to differentiate disordered structures. Moreover, including optical properties may eventually lead to a deep learning tool that could predict process parameters for PhG with optimized optical properties.

Based on the analysis by SEM and DCNN, films with different morphologies were selected and infiltrated with $\mathrm{TiO}_{2}$ via ALD to fabricate surface-templated inverse photonic glasses. The inverted films produced with 95 and 100 wt \% $\mathrm{NR}_{3}{ }^{+}$-functionalized particles presented intense blue structural coloration; however, cracking and strong delamination were observed. Since the film quality is an important aspect to be considered in the fabrication of high-quality structural colored films over large areas, the inverse photonic glass from the mix with $35 \mathrm{wt} \% \mathrm{NR}_{3}{ }^{+}$-functionalized particles showed high film stability while also exhibiting blue structural coloration. Therefore, the heteroaggregation process developed in this work provides new opportunities to produce high-quality photonic glass films over large areas. Moreover, the inverted $\mathrm{TiO}_{2}$ structures enable the application of the films in hightemperature fields.

\section{ASSOCIATED CONTENT}

\section{Supporting Information}

The Supporting Information is available free of charge at https://pubs.acs.org/doi/10.1021/acsami.1c01392.

Zeta potential results, enlarged FFTs, contact angle measurement results, SEM images of film cross sections, sample weight over time plots of all films, height profiles of all films, DCNN architecture, specular reflection spectra of all films, and diffuse reflection spectra of all films (PDF)

\section{AUTHOR INFORMATION}

\section{Corresponding Author}

Kaline P. Furlan - Institute of Advanced Ceramics, Hamburg University of Technology, 21073 Hamburg, Germany; ○ orcid.org/0000-0003-4032-2795; Email: kaline.furlan@ tuhh.de

\section{Authors}

Yen Häntsch - Institute of Advanced Ceramics, Hamburg University of Technology, 21073 Hamburg, Germany

Guoliang Shang - Institute of Optical and Electronic Materials, Hamburg University of Technology, 21073 Hamburg, Germany; ○ orcid.org/0000-0002-8872-8789

Bo Lei - Department of Materials Science and Engineering, Carnegie Mellon University, Pittsburgh, Pennsylvania 15213, United States

Benedikt Winhard - Institute of Advanced Ceramics, Hamburg University of Technology, 21073 Hamburg, Germany

Alexander Petrov - Institute of Optical and Electronic Materials, Hamburg University of Technology, 21073 Hamburg, Germany; ITMO University, 197101 St. Petersburg, Russia

Manfred Eich - Institute of Optical and Electronic Materials, Hamburg University of Technology, 21073 Hamburg, Germany; Institute of Materials Research, HelmholtzZentrum Geesthacht, 21502 Geesthacht, Germany 
Elizabeth Holm - Department of Materials Science and Engineering, Carnegie Mellon University, Pittsburgh, Pennsylvania 15213, United States

Gerold A. Schneider - Institute of Advanced Ceramics, Hamburg University of Technology, 21073 Hamburg, Germany; (1) orcid.org/0000-0001-5780-6249

Complete contact information is available at: https://pubs.acs.org/10.1021/acsami.1c01392

\section{Author Contributions}

K.P.F was responsible for the conception, supervision, and coordination of the study. K.P.F. and Y.H. were responsible for the production of the photonic glasses. Y.H. characterized the colloidal suspensions, and both K.P.F. and Y.H. performed the SEM analysis of the photonic structures. G.S. measured and analyzed the optical properties. B.W. performed the profilometry measurements and analysis. B.L. and E.H. were responsible for the DCNN image analysis. All authors contributed to the results' discussion, as well as manuscript preparation, and have given approval to its final version.

\section{Notes}

The authors declare no competing financial interest.

\section{ACKNOWLEDGMENTS}

This study was funded by the Deutsche Forschungsgemeinschaft (DFG, German Research Foundation)-projektnummer 192346071-SFB 986, projects C4 and C2. K.P.F. also gratefully acknowledges Prof. Robert Blick and Dr. Robert Zierold for the access to the profilometer of the Center for Hybrid Nanostructures at the University of Hamburg, Germany.

\section{REFERENCES}

(1) Lee, H. S.; Kubrin, R.; Zierold, R.; Petrov, A. Y.; Nielsch, K.; Schneider, G. A.; Eich, M. Thermal Radiation Transmission and Reflection Properties of Ceramic 3D Photonic Crystals. J. Opt. Soc. Am. B 2012, 29, 450.

(2) Shklover, V.; Braginsky, L.; Witz, G.; Mishrikey, M.; Hafner, C. High-Temperature Photonic Structures. Thermal Barrier Coatings, Infrared Sources and Other Applications. J. Comput. Theor. Nanosci. 2008, 5, 862-893.

(3) Shang, G.; Maiwald, L.; Renner, H.; Jalas, D.; Dosta, M.; Heinrich, S.; Petrov, A.; Eich, M. Photonic Glass for High Contrast Structural Color. Sci. Rep. 2018, 8, 7804.

(4) Caixeiro, S.; Gaio, M.; Marelli, B.; Omenetto, F. G.; Sapienza, R. Silk-Based Biocompatible Random Lasing. Adv. Opt. Mater. 2016, 4, 998-1003.

(5) Shang, G.; Eich, M.; Petrov, A. Photonic Glass Based Structural Color. APL Photonics 2020, 5, 060901.

(6) Ballato, J.; Dimaio, J.; James, A.; Gulliver, E. Photonic Band Engineering through Tailored Microstructural Order. Appl. Phys. Lett. 1999, 75, 1497-1499.

(7) García, P. D.; Sapienza, R.; Blanco, Á.; López, C. Photonic Glass: A Novel Random Material for Light. Adv. Mater. 2007, 19, 25972602.

(8) García, P. D.; Sapienza, R.; López, C. Photonic Glasses: A Step Beyond White Paint. Adv. Mater. 2010, 22, 12-19.

(9) Espinha, A.; Ibisate, M.; Blanco, A.; López, C. Engineering the Light-Transport Mean Free Path in Silica Photonic Glasses. Part. Part. Syst. Charact. 2016, 33, 352-357.

(10) Eurov, D. A.; Kurdyukov, D. A.; Trofimova, E. Y.; Yakovlev, S. A.; Sharonova, L. V.; Shvidchenko, A. V.; Golubev, V. G. Preparation of Colloidal Films with Different Degrees of Disorder from Monodisperse Spherical Silica Particles. Phys. Solid State 2013, 55, $1718-1724$.
(11) Harun-Ur-Rashid, M.; Bin Imran, A.; Seki, T.; Ishii, M.; Nakamura, H.; Takeoka, Y. Angle-independent Structural Color in Colloidal Amorphous Arrays. ChemPhysChem 2010, 11, 579-583.

(12) Kim, S.-H.; Magkiriadou, S.; Rhee, D. K.; Lee, D. S.; Yoo, P. J.; Manoharan, V. N.; Yi, G.-R. Inverse Photonic Glasses by Packing Bidisperse Hollow Microspheres with Uniform Cores. ACS Appl. Mater. Interfaces 2017, 9, 24155-24160.

(13) Forster, J. D.; Noh, H.; Liew, S. F.; Saranathan, V.; Schreck, C. F.; Yang, L.; Park, J.-G.; Prum, R. O.; Mochrie, S. G. J.; O’Hern, C. S.; Cao, H.; Dufresne, E. R. Biomimetic Isotropic Nanostructures for Structural Coloration. Adv. Mater. 2010, 22, 2939-2944.

(14) Zhang, Y.; Dong, B.; Chen, A.; Liu, X.; Shi, L.; Zi, J. Photonics: Using Cuttlefish Ink as an Additive to Produce Non-iridescent Structural Colors of High Color Visibility. Adv. Mater. 2015, 27, 4666.

(15) Chen, G.; Yi, B.; Huang, Y.; Liang, Q.; Shen, H. Development of Bright and Low Angle Dependence Structural Colors from OrderDisorder Hierarchical Photonic Structure. Dyes Pigm. 2019, 161, 464-469.

(16) do Rosário, J. J.; Dyachenko, P. N.; Kubrin, R.; Pasquarelli, R. M.; Petrov, A. Y.; Eich, M.; Schneider, G. A. Facile Deposition of YSZ-Inverse Photonic Glass Films. ACS Appl. Mater. Interfaces 2014, 6, 12335-12345.

(17) Jia, Y.; Duran, C.; Hotta, Y.; Sato, K.; Watari, K. Macroporous $\mathrm{ZrO}_{2}$ Ceramics Prepared from Colloidally Stable Nanoparticles Building Blocks and Organic Templates. J. Colloid Interface Sci. 2005, 291, 292-295.

(18) Jia, Y.; Duran, C.; Hotta, Y.; Sato, K.; Watari, K. The Effect of Polyelectrolyte on Fabrication of Macroporous $\mathrm{ZrO}_{2}$ Ceramics. J. Mater. Sci. 2005, 40, 2903-2909.

(19) Sakka, Y.; Tang, F.; Fudouzi, H.; Uchikoshi, T. Fabrication of Porous Ceramics with Controlled Pore Size by Colloidal Processing. J. Am. Ceram. Soc. 2005, 6, 915-920.

(20) Tang, F. Preparation of Macroporous Titania from Nanoparticle Building Blocks and Polymer Templates. Scr. Mater. 2003, 49, 735-740.

(21) Tang, F.; Fudouzi, H.; Sakka, Y. Fabrication of Macroporous Alumina with Tailored Porosity. J. Am. Ceram. Soc. 2003, 86, 20502054.

(22) Tang, F.; Fudouzi, H.; Uchikoshi, T.; Sakka, Y. Preparation of Porous Materials with Controlled Pore Size and Porosity. J. Eur. Ceram. Soc. 2004, 24, 341-344.

(23) Tang, F.; Uchikoshi, T.; Ozawa, K.; Sakka, Y. Effect of Polyethylenimine on the Dispersion and Electrophoretic Deposition of Nano-sized Titania Aqueous Suspensions. J. Eur. Ceram. Soc. 2006, 26, 1555-1560.

(24) Tang, F.; Uchikoshi, T.; Sakka, Y. A Practical Technique for the Fabrication of Highly Ordered Macroporous Structures of Inorganic Oxides. Mater. Res. Bull. 2006, 41, 268-273.

(25) Cerbelaud, M.; Videcoq, A.; Abélard, P.; Pagnoux, C.; Rossignol, F.; Ferrando, R. Heteroaggregation between $\mathrm{Al}_{2} \mathrm{O}_{3}$ Submicrometer Particles and $\mathrm{SiO}_{2}$ Nanoparticles: Experiment and Simulation. Langmuir 2008, 24, 3001-3008.

(26) Cao, H.; Zhang, L.; Wu, L.; Kong, X. Z. Characterization of Heterocoagulation with Oppositely Charged Polymer Colloid Particles through Online Tracking of Light Transmittance. ACS Appl. Mater. Interfaces 2016, 8, 29136-29147.

(27) Leib, E. W.; Pasquarelli, R. M.; do Rosário, J. J.; Dyachenko, P. N.; Döring, S.; Puchert, A.; Petrov, A. Y.; Eich, M.; Schneider, G. A.; Janssen, R.; Weller, H.; Vossmeyer, T. Yttria-stabilized Zirconia Microspheres: Novel Building Blocks for High-Temperature Photonics. J. Mater. Chem. C 2016, 4, 62-74.

(28) Kubrin, R.; Pasquarelli, R. M.; Waleczek, M.; Lee, H. S.; Zierold, R.; do Rosário, J. J.; Dyachenko, P. N.; Montero Moreno, J. M.; Petrov, A. Y.; Janssen, R.; Eich, M.; Nielsch, K.; Schneider, G. A. Bottom-up Fabrication of Multilayer Stacks of 3D Photonic Crystals from Titanium Dioxide. ACS Appl. Mater. Interfaces 2016, 8, 1046610476.

(29) Furlan, K. P.; Pasquarelli, R. M.; Krekeler, T.; Ritter, M.; Zierold, R.; Nielsch, K.; Schneider, G. A.; Janssen, R. Highly Porous 
$\alpha-\mathrm{Al}_{2} \mathrm{O}_{3}$ Ceramics Obtained by Sintering Atomic Layer Deposited Inverse Opals. Ceram. Int. 2017, 43, 11260-11264.

(30) Furlan, K. P.; Krekeler, T.; Ritter, M.; Blick, R.; Schneider, G. A.; Nielsch, K.; Zierold, R; Janßen, R. Low-Temperature Mullite Formation in Ternary Oxide Coatings Deposited by ALD for HighTemperature Applications. Adv. Mater. Interfaces 2017, 4 (). DOI: DOI: $10.1002 /$ admi.201700912.

(31) Hunt, N.; Jardine, R.; Bartlett, P. Superlattice Formation in Mixtures of Hard-Sphere Colloids. Phys. Rev. E: Stat. Phys., Plasmas, Fluids, Relat. Interdiscip. Top. 2000, 62, 900-913.

(32) Lee, G. H.; Kim, J. B.; Choi, T. M.; Lee, J. M.; Kim, S. H. Structural Coloration with Nonclose-Packed Array of Bidisperse Colloidal Particles. Small 2019, 15, No. e1804548.

(33) Lin, W.; Kobayashi, M.; Skarba, M.; Mu, C.; Galletto, P.; Borkovec, M. Heteroaggregation in Binary Mixtures of Oppositely Charged Colloidal Particles. Langmuir 2006, 22, 1038-1047.

(34) Mukherjee, S.; Saha, A.; Santra, P. K.; Sengupta, S.; Sarma, D. D. Beyond the "Coffee Ring": Re-entrant Ordering in an Evaporationdriven Self-Assembly in a Colloidal Suspension on a Substrate. J. Phys. Chem. B 2014, 118, 2559-2567.

(35) Shang, G.; Furlan, K. P.; Janßen, R.; Petrov, A.; Eich, M. Surface Templated Inverse Photonic Glass for Saturated Blue Structural Color. Opt. Express 2020, 28, 7759-7770.

(36) Holm, E. A.; Cohn, R.; Gao, N.; Kitahara, A. R.; Matson, T. P.; Lei, B.; Yarasi, S. R. Overview: Computer Vision and Machine Learning for Microstructural Characterization and Analysis. Metall. Mater. Trans. A 2020, 51, 5985-5999.

(37) DeCost, B. L.; Francis, T.; Holm, E. A. Exploring the Microstructure Manifold: Image Texture Representations Applied to Ultrahigh Carbon Steel Microstructures. Acta Mater. 2017, 133, 3040.

(38) DeCost, B. L.; Lei, B.; Francis, T.; Holm, E. A. High Throughput Quantitative Metallography for Complex Microstructures Using Deep Learning: A Case Study in Ultrahigh Carbon Steel. Microsc. Microanal. 2019, 25, 21-29.

(39) Ling, J.; Hutchinson, M.; Antono, E.; DeCost, B.; Holm, E. A.; Meredig, B. Building Data-driven Models with Microstructural Images: Generalization and Interpretability. Mater. Discovery 2017, $10,19-28$.

(40) Simonyan, K.; Zisserman, A. Very Deep Convolutional Networks for Large-Scale Image Recognition. International Conference on Learning Representations 2015. 2015, arXiv:1409.1556, 114.

(41) Russakovsky, O.; Deng, J.; Su, H.; Krause, J.; Satheesh, S.; Ma, S.; Huang, Z.; Karpathy, A.; Khosla, A.; Bernstein, M.; Berg, A. C.; Fei-Fei, L. ImageNet Large Scale Visual Recognition Challenge. Int. J. Comput. Vis. 2015, 115, 211-252.

(42) van der Maaten, L.; Hinton, G. Visualizing Data using t-SNE. J. Mach. Learn. Res. 2008, 9, 2579-2605.

(43) He, K.; Zhang, X.; Ren, S.; Sun, J. Deep Residual Learning for Image Recognition. 2016 IEEE Conference on Computer Vision and Pattern Recognition (CVPR); IEEE, 2016; pp 770-778.

(44) Shim, S.-E.; Cha, Y.-J.; Byun, J.-M.; Choe, S. Size Control of Polystyrene Beads by Multistage Seeded Emulsion Polymerization. J. Appl. Polym. Sci. 1999, 71, 2259-2269.

(45) Jones, G. D. Chloromethylation of Polystyrene. Ind. Eng. Chem. 1952, 44, 2686-2693.

(46) Piret, F.; Su, B.-L. Effects of $\mathrm{pH}$ and Ionic Strength on the SelfAssembly of Silica Colloids to Opaline Photonic Structures. Chem. Phys. Lett. 2008, 457, 376-380.

(47) Bhardwaj, R.; Fang, X.; Somasundaran, P.; Attinger, D. SelfAssembly of Colloidal Particles from Evaporating Droplets: Role of DLVO Interactions and Proposition of a Phase Diagram. Langmuir 2010, 26, 7833-7842.

(48) Carré, A.; Lacarrière, V.; Birch, W. Molecular Interactions between DNA and an Aminated Glass Substrate. J. Colloid Interface Sci. 2003, 260, 49-55.
(49) Oh, S. H.; Scheu, C.; Wagner, T.; Rühle, M. Control of Bonding and Epitaxy at Copper/Sapphire Interface. Appl. Phys. Lett. 2007, 91, 141912.

(50) Yang, D.; Krasowska, M.; Sedev, R.; Ralston, J. The Unusual Surface Chemistry of $\alpha-\mathrm{Al}_{2} \mathrm{O}_{3}$ (0001). Phys. Chem. Chem. Phys. 2010, 12, 13724-13729.

(51) Jiang, P.; Bertone, J. F.; Hwang, K. S.; Colvin, V. L. SingleCrystal Colloidal Multilayers of Controlled Thickness. Chem. Mater. 1999, 11, 2132-2140.

(52) Romanov, S. G.; Orlov, S.; Ploss, D.; Weiss, C. K.; Vogel, N.; Peschel, U. Engineered Disorder and Light Propagation in a Planar Photonic Glass. Sci. Rep. 2016, 6, 27264.

(53) Yan, Q.; Gao, L.; Sharma, V.; Chiang, Y.-M.; Wong, C. C. Particle and Substrate Charge Effects on Colloidal Self-Assembly in a Sessile Drop. Langmuir 2008, 24, 11518-11522.

(54) Huang, Y.; Zhou, J.; Su, B.; Shi, L.; Wang, J.; Chen, S.; Wang, L.; Zi, J.; Song, Y.; Jiang, L. Colloidal Photonic Crystals with Narrow Stopbands Assembled from Low-adhesive Superhydrophobic Substrates. J. Am. Chem. Soc. 2012, 134, 17053-17058.

(55) Wang, L.; Zhao, X. S. Fabrication of Crack-Free Colloidal Crystals Using a Modified Vertical Deposition Method. J. Phys. Chem. C 2007, 111, 8538-8542.

(56) Hatton, B.; Mishchenko, L.; Davis, S.; Sandhage, K. H.; Aizenberg, J. Assembly of Large-Area, Highly Ordered, Crack-free Inverse Opal Films. Proc. Natl. Acad. Sci. U.S.A. 2010, 107, 1035410359.

(57) Tirumkudulu, M. S.; Russel, W. B. Cracking in Drying Latex Films. Langmuir 2005, 21, 4938-4948.

(58) Phillips, K. R.; Zhang, C. T.; Yang, T.; Kay, T.; Gao, C.; Brandt, S.; Liu, L.; Yang, H.; Li, Y.; Aizenberg, J.; Li, L. Fabrication of Photonic Microbricks via Crack Engineering of Colloidal Crystals. Adv. Funct. Mater. 2019, 30, 1908242.

(59) Campos, H. G.; Furlan, K. P.; Garcia, D. E.; Blick, R.; Zierold, R.; Eich, M.; Hotza, D.; Janssen, R. Effects of Processing Parameters on 3D Structural Ordering and Optical Properties of Inverse Opal Photonic Crystals Produced by Atomic Layer Deposition. Int. J. Ceram. Eng. Sci. 2019, 1, 68-76.

(60) Häntsch, Y.; Shang, G.; Petrov, A.; Eich, M.; Schneider, G. A. YSZ Hollow Sphere Photonic Glasses: Tailoring Optical Properties for Highly Saturated Non-Iridescent Structural Coloration. Adv. Opt. Mater. 2019, 7, 1900428.

(61) Shang, G.; Furlan, K. P.; Zierold, R.; Blick, R. H.; Janßen, R.; Petrov, A.; Eich, M. Transparency Induced in Opals via Nanometer Thick Conformal Coating. Sci. Rep. 2019, 9, 11379.

(62) Furlan, K. P.; Larsson, E.; Diaz, A.; Holler, M.; Krekeler, T.; Ritter, M.; Petrov, A. Y.; Eich, M.; Blick, R.; Schneider, G. A.; Greving, I.; Zierold, R.; Janßen, R. Photonic Materials for HighTemperature Applications: Synthesis and Characterization by X-ray Ptychographic Tomography. Appl. Mater. Today. 2018, 13, 359-369.

(63) Moehl, T.; Suh, J.; Sévery, L.; Wick-Joliat, R.; Tilley, S. D. Investigation of (Leaky) ALD $\mathrm{TiO}_{2}$ Protection Layers for WaterSplitting Photoelectrodes. ACS Appl. Mater. Interfaces 2017, 9, 43614-43622.

(64) Iwata, M.; Teshima, M.; Seki, T.; Yoshioka, S.; Takeoka, Y. BioInspired Bright Structurally Colored Colloidal Amorphous Array Enhanced by Controlling Thickness and Black Background. Adv. Mater. 2017, 29, 1605050

(65) Pasquarelli, R. M.; Lee, H. S.; Kubrin, R.; Zierold, R.; Petrov, A. Y.; Nielsch, K.; Schneider, G. A.; Eich, M.; Janssen, R. Enhanced Structural and Phase Stability of Titania Inverse Opals. J. Eur. Ceram. Soc. 2015, 35, 3103-3109.

(66) Polymer Data Handbook, 2nd ed.; Mark, J. E., Ed.; Oxford University Press, 2009. 